\title{
Management and Treatment Guidelines for Sepsis in Pediatric Patients
}

\author{
Nidal El-wiher ${ }^{1}$, Timothy T. Cornell ${ }^{1}$, Niranjan Kissoon ${ }^{2}$ and Thomas P. Shanley*,1 \\ ${ }^{I}$ Division of Critical Care Medicine, C.S. Mott Children's Hospital at the University of Michigan, Ann Arbor, MI, USA; \\ ${ }^{2}$ Division of Critical Care Medicine, British Columbia Children's Hospital, University of British Columbia
}

\begin{abstract}
Sepsis remains one of the leading causes of morbidity and mortality in children despite improved understanding of the pathophysiology leading to better clinical management and survival. Recent studies have identified several areas that must be addressed by the clinician in order to continue to impact the morbidity and mortality associated with sepsis. In this review, we discuss the evidence in several of these areas including initial resuscitation, pathogen eradication, maintenance of oxygen delivery, and directed modifiers of the inflammatory response. Our overall goal is to provide the bedside clinician with an updated systematic approach to treat sepsis in children.
\end{abstract}

Keywords: Sepsis, pediatrics, guidelines, management.

\section{INTRODUCTION}

Sepsis, defined as the systemic inflammatory response triggered by a known or highly suspected pathogen remains among the major causes of morbidity and mortality in children worldwide. The advent of modern intensive care technology, pharmacology and expertise coupled with an improved understanding of the pathophysiology of sepsis has led to significant improvements in mortality in some areas of the world. For instance, in some areas such as the United States, the mortality rate of severe sepsis in children has been declining over the last decade from $\sim 10.3 \%$ to $9 \%$ as reported by Watson and colleagues studies $[1,2]$ to $4.2 \%$ in the most recent study done by Odetola and colleagues [3]. This decline of mortality rate has been observed even in the context of an increase the absolute number of cases with severe sepsis (rising $\sim 13 \%$ per year) in children [2]. A possible explanation for this observation could be dedicated teams with sufficient resources and consistent adherence to guidelines of sepsis management [4] among the pediatric population. However, despite these tremendous advances, the morbidity and mortality attributed to sepsis remain unacceptably high as severe sepsis is now classified as one of the leading causes of death in children with an estimated 4,400 deaths occurring annually in the United States alone [2]. Furthermore, pediatric sepsis accounts for an estimated annual health care cost of $\$ 2$ billion in the United States [3]. Perhaps even more important, the worldwide burden of sepsis, particularly in underdeveloped and developing countries, has not been quantified in terms of health care dollars, but its costs in terms of human suffering and lives lost are substantially higher than in the developed regions of the world [5]. Thus, while improved mechanistic understanding obtained via basic and translational science will continue to identify novel therapeutic targets and

*Address correspondence to this author at the C.S. Mott Children's Hospital, F-6892, University of Michigan Medical School, 1500 East Medical Center Drive, Ann Arbor, MI 48109, USA;

Tel: 734-764-5302; Fax: 734-647-5624; E-mail: tshanley@med.umich.edu approaches, an emphasis needs to remain on managing pediatric sepsis with the best clinical practice. Such practice needs to not only be supported by strong evidence but also consider modifications for care providers in countries with limited resources. As reviewed below, therapeutic approaches to sepsis remain predominantly supportive but are founded on fundamental physiologic principles that largely define critical care medicine and entail four important goals: initial resuscitation, pathogen eradication, maintenance of oxygen delivery, and more specifically directed modifiers of the inflammatory response. In the near future, emerging data from genetic, genomic and other translation studies in pediatrics will undoubtedly identify targets for newer therapies.

\section{INITIAL RESUSCITATION}

The primary goals of therapy in the first hours following clinical presentation are to maintain oxygenation and ventilation and achieve normal perfusion. Important clinical parameters that are reflective of "normal perfusion" include: capillary refill $<2$ seconds, normal pulses with no differential between the quality of peripheral and central pulses, warm extremities, normal mental status, normal blood pressure for age and appropriate urine output for age. Early medical responders (e.g. EMS and transport personnel, ED staff) should ensure a patent airway and appropriate protective airway reflexes are present, especially if there is any alteration in mental status. They should make a rapid assessment of the work of breathing, air exchange, and measure oxygen saturation non-invasively when pulse oximetry is available. When any degree of distress or abnormal respiratory function is noted, the patient should be placed on $100 \%$ oxygen to ensure maximal oxygen saturation. In the presence of metabolic acidosis related to septic shock, the work of breathing associated with the compensatory respiratory alkalosis can be substantial and contribute, along with fever, to a significant portion of oxygen consumption. In addition, myocardial dysfunction, which is often present in children with sepsis, can be partially ameliorated by the application of positive pressure 
ventilation by achieving a decrease in afterload (provided impairment of preload is not too great). Therefore, early semi-elective intubation and positive pressure mechanical ventilatory support should be strongly considered in this setting. This approach is supported in part by the clinical practice position article from a group that reported low mortality $(\sim 5 \%)$ in meningococcal septic shock by intubating and mechanically ventilating all children who required $>40$ $\mathrm{cc}$ 's $/ \mathrm{kg}$ volume resuscitation [6]. Often, the combination of increased lung vascular permeability with aggressive fluid resuscitation that is necessary to restore intravascular volume and maintain organ perfusion pressure (defined as the difference between the mean arterial pressure minus the mean venous pressure) contributes to the development of pulmonary edema. In children, the related changes in lung compliance and loss of functional residual capacity tremendously increase the work of breathing ultimately leading to respiratory failure that necessitates endotracheal intubation and mechanical ventilatory support. The presence of substantially increased work of breathing or alternatively, ominous signs of hypoventilation, impaired mental status, or obtundation are all indications for instituting mechanical ventilatory support which holds additional benefit in decreasing the overall oxygen consumption, especially when combined with sedation and paralysis. Because of the often dramatic effect the initiation of positive pressure ventilation can have on the hypovolemic patient in decreasing preload and thus, cardiac output, it is strongly suggested that intravascular volume depletion be corrected prior to instituting positive pressure ventilation and that additional volume expansion be readily available in this setting.

\section{Fluid Resuscitation}

Following airway and breathing assessment and management, circulation is the next target to assess and manage. An increasingly recognized approach is an effort to rapidly stabilize the hemodynamics using early, goaldirected therapy (EGDT). This approach grew out of a now seminal study in adults conducted by Rivers and coworkers [4]. These investigators reported the outcomes from a randomized, controlled trial in 203 patients with severe sepsis and septic shock who received early--within 6 hours of clinical presentation--goal-directed therapy. Investigators used a well-defined pathway involving adjustments in preload, afterload, contractility and oxygen carrying capacity to optimize oxygen delivery as reflected by an improvement/normalization of superior vena cava hemoglobin saturation. In this study, EGDT was associated with decreased mortality at both 28 and 60 days and in hospital mortality was reduced from $46.5 \%$ in the group assigned to standard therapy to $30.5 \%$ in EGDT [4]. Driven in part by this apparent success of early, aggressive resuscitation, current guidelines for the management of pediatric shock continue to emphasize the need for early and adequate fluid resuscitation within the first 15 minutes (see Fig. 1) [7]. After achieving adequate intravenous access (preferably via a central vein), the goal is to initiate fluid resuscitation with $20 \mathrm{cc} / \mathrm{kg}$ of isotonic fluid as quickly as possible targeting up to $60 \mathrm{cc} / \mathrm{kg}$ and perhaps more within the first 15 to 60 minutes.

In striking parallel to the Rivers study, de Oliveira and his group in Brazil conducted a similar randomized controlled trial in a pediatric population. In this cohort of 102 children with severe sepsis, similar early goal-directed therapy was implemented to achieve and maintain superior vena caval oxygen $\left(\mathrm{SVC} \mathrm{O}_{2}\right)$ saturation $>70 \%$ [8]. In the study 28-day mortality was significantly lower in the intervention group $(11.8 \%$ vs. $39.2 \%)$ with significantly fewer new organ dysfunctions. Interestingly, SVC $\mathrm{O}_{2}$ saturation on admission carried a prognostic value, as mortality rate was significantly higher in patients who presented with $\mathrm{SVC} \mathrm{O}_{2}$ saturation $<70 \%(41.9 \%$ vs. $24.3 \%$; $\mathrm{OR}=3.04(1.43-8.79) ; \mathrm{p}=0.006)$. Though only a single center study and mortality rates trended higher in both groups as compared to rates reported from other recent pediatric sepsis studies, these results support the concept that a strategy of achieving early restoration of adequate organ perfusion pressure, espoused by early goal directed therapy, may improve outcome in children with septic shock.

\section{Choice of Fluid Resuscitation}

There is still controversy surrounding the question as to whether crystalloid or colloid fluid is superior for volume resuscitation. The adult SAFE trial demonstrated no significant difference in 28 day mortality between the $4 \%$ albumin and normal saline groups suggesting crystalloid is a cheaper yet equally effective option [9]. As there is no pediatric literature to support colloid over crystalloid, most current practitioners tend to choose crystalloid as it is more readily available and less expensive, with the exception being the cardiac intensive care unit population in whom 5\% albumin continues to be a common choice for volume resuscitation. It may be that the underlying cause of hypotension may be a critical factor in determining the clinical response to one or the other. For example, Maitland et al. [10] reported a reduced mortality rate (from $18 \%$ to 3.6 $\%$ ) in children with severe malaria and metabolic acidosis when $4.5 \%$ albumin was used for fluid resuscitation rather than normal saline. Thus, while it remains at the discretion of the care provider to choose the type of fluid for resuscitation, crystalloids are valid choices given the expense, ease of availability and effectiveness.

While following clinical exam for signs of overly aggressive volume resuscitation (new onset of rales, increased work of breathing, development of a gallop, or hepatomegaly), fluid should be administered quickly with attention to responses in heart rate, urine output, capillary refill time and level of consciousness. Blood transfusion should be used only to maintain adequate $\mathrm{SVC} \mathrm{O}_{2}$ saturation $>70 \%$ when the $\mathrm{Hgb}$ is low (usually less than $10 \mathrm{gm} / \mathrm{dl}$ ). Fresh frozen plasma transfusions are used only to correct an underlying coagulopathy associated with either active bleeding or a high risk of bleeding. In each case of blood product transfusion, attention must be paid to maintain a normal ionized calcium level which can be reduced by citrate used for blood product storage. If adequate resuscitation endpoints are not reached by fluid resuscitation alone- $\mathrm{a}$ socalled state of "fluid refractory shock"--the patient will need vasoactive/inotropic agents (Fig. 1).

\section{Inotropic/Vasoactive Agents}

\section{Dopamine}

Dopamine remains the most common first choice of inotropic agents for patients with fluid-refractory shock. 


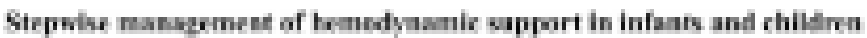

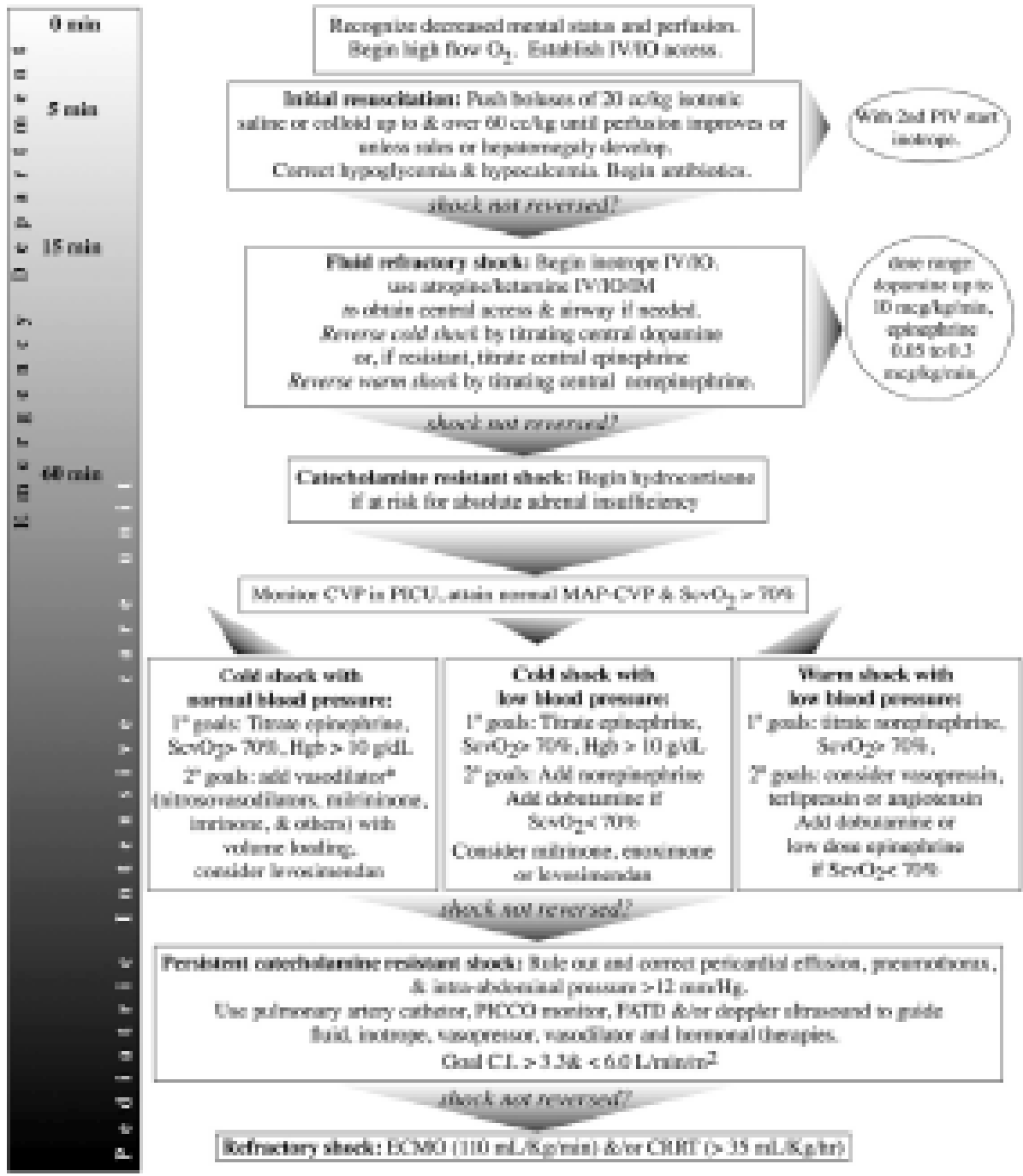

Fig. (1). Clinical Practice Parameters for Septic Shock [7].

Dopamine possesses dose-dependent agonist effects on dopaminergic and adrenergic ( $\alpha$ and $\beta$ ) receptors. Thus, dopamine provides inotropic support via $\beta$-adrenergic stimulation in the general dosing range of $5-10 \mu \mathrm{g} / \mathrm{kg} / \mathrm{min}$ and vasopressor activity via $\alpha$-adrenergic stimulation in the higher dosing range $(\sim 10-20 \mu \mathrm{g} / \mathrm{kg} / \mathrm{min})$. More recently, dopamine has lost some favor as the first line inotropic agent because of a number of reported and theoretic adverse effects. Dopamine functionally suppresses neutrophils by attenuating the chemoattractant effect of interleukin-8 [11]. It also interferes with the neuroendocrine system by suppressing the release of many anterior pituitary hormones, including growth hormone, TSH and prolactin. Because prolactin typically enhances monocytes, B-cell and T-cell responses and may prevent their programmed cell death (apoptosis), the use of dopamine may aggravate immune dysfunction and increase susceptibility to infection [11, 12]. Therefore, clinicians are increasingly choosing low-dose epinephrine to achieve $\beta$-adrenergic-mediated inotropic support as a first line therapy for fluid refractory shock (see below). When the hemodynamic resuscitation end-points are not achieved with adequate fluid and dopamine/low-dose epinephrine administration, further management of this socalled state of "fluid-refractory, dopamine-resistant shock" is dictated by the clinical context.

\section{Epinephrine/Norepinephrine}

The decision of which agent to add in the setting of fluidrefractory, dopamine-resistant shock is based on the underlying cause of cardiovascular compromise and the presenting hemodynamic profile. In the pediatric population it more common to have low cardiac output with increased systemic vascular resistance as compared to adults who most often present with high cardiac output and low systemic vascular resistance [13]. As a result, low-dose epinephrine is chosen as the second line inotropic agent most often. Epinephrine provides inotropic support via $\beta 1$ receptor stimulation and modest vasodilatation via $\beta 2$ receptor stimulation when administered at low concentrations $(0.02$ $0.3 \mu \mathrm{g} / \mathrm{kg} / \mathrm{min}$ ). Though epinephrine possesses these beneficial cardiovascular effects, it too possesses some adverse properties. Epinephrine stimulates gluconeogenesis and glycogenolysis and inhibits the action of insulin, leading to an adverse profile of dysregulated serum glucose which 
may impact outcomes. Epinephrine also increases the production of lactate independent of organ perfusion as seen in lactic acidosis type $B_{2}$ [14] which makes following lactate as a marker for organ perfusion a less reliable marker to trend in children with septic shock managed with epinephrine.

In the setting of low systemic vascular resistance (SVR), higher concentrations of epinephrine $(>0.3 \mu \mathrm{g} / \mathrm{kg} / \mathrm{min})$ are used to provide vasopressor effect via $\alpha 1$ stimulation. Furthermore, given the observation that the SVR is often high (clinically presenting as "cold" shock), vasodilator therapy should be considered in the adequately volume resuscitated, relatively normotensive patient who remains mottled with evidence of end-organ malperfusion (e.g. elevated lactate, decreased urine output, delayed capillary refill). In the less common hemodynamic state in pediatric severe sepsis of high cardiac output and low SVR, a vasopressor such as norepinephrine, which provides vasoconstriction via $\alpha 1$ receptor stimulation is most commonly used. Norepinephrine also provides some minimal inotropic support via its less predominant $\beta 1$ receptor stimulation.

\section{Milrinone}

Milrinone is a type III phosphodiesterase (PDE) inhibitor that may have a synergistic effect with $\beta$-adrenergic agonists since the latter agents stimulate intracellular production of cAMP while the PDE inhibitors further increase intracellular cAMP by blocking its degradation. Since the PDE inhibitors do not depend on a receptor mechanism, they maintain their action even when the $\beta$-adrenergic receptors are downregulated or have reduced functional responsiveness as may be the case in sepsis. A caveat to its use is that it possesses a long elimination half-life, which is more pronounced in patients with renal dysfunction, so that milrinone should be discontinued at the first sign of hypotension or excessively diminished systemic vascular resistance. Because of this, some clinicians prefer to employ a much shorter-acting, more easily titratable vasodilator (e.g. nitroprusside) to decipher the clinical response to afterload-reduction, which can be immediately discontinued in the event of unacceptable hypotension.

\section{Vasopressin}

This natural hormone works at 3 different receptors: $V 1$ located on vascular smooth cells which mediate vasoconstriction and enhancement of prostaglandin release; $V 2$ found on the distal convoluted tubule and medulla collecting ducts which mediate an anti-diuretic effect; and $V 3$ located on the anterior hypophysis and pancreatic islets which facilitate the release of ACTH and insulin secretion [15]. In septic shock, the vasopressin concentrations increase to $200-300 \mathrm{pg} / \mathrm{ml}$ but after one hour, the neurohypophysial stores of vasopressin are depleted and plasma concentration may fall dramatically (to an approximate mean if $\sim 30 \mathrm{pg} / \mathrm{ml}$ ) [16]. In states of sepsis there is also down-regulation of vasopressin receptors. Thus, infusion of low-dose vasopressin has been reported to increase blood pressure and urine output. However, its use comes with risks in that it may decrease cardiac output (because of the increased afterload without any concomitant inotropic effect), contribute to intestinal ischemia, and potentially cause skin necrosis. Its use has even been associated with cardiac arrest when used at especially high doses $(>0.4 \mathrm{u} / \mathrm{min})$. As a result, with the lack of sufficient data to direct care, the use of vasopressin in pediatric sepsis seems to be most commonly restricted to children with significant vasodilatory shock and should be used with caution in all cases.

It is important to note that resuscitation end-points targeted in resource-rich health care environments (e.g. SVC $\mathrm{O} 2$ saturation, central venous pressure (CVP), echocardiographic determination of cardiac output (CO)) are not available to intensive care personnel in many developing parts of the world. As a result, these practitioners rely on clinical therapeutic end-points: normalization of heart rate, capillary refill time 2 secs $<$, strong peripheral pulses, warm extremities, normal blood pressure, return to baseline mental status and normal respiratory rate. There are clearly limitations to this approach which have led to the adjuvant use of technology-based assessments such as arterial blood gas with lactate determination, CVP measurements and central vein hemoglobin saturation sampling, and noninvasive $\mathrm{CO}$ determination. As global initiatives link intensivists across a spectrum of health care environments, more information is needed to define the role of such technology-based measurements in both directing care and impacting mortality in pediatric sepsis in contrast to settings without access to such resources.

\section{ELIMINATION OF PATHOGEN}

Prompt initiation of appropriate anti-microbial therapy against the causative pathogens has been shown to be one of the most important predictors of outcome. In a seminal study of over 1100 patients, the provision of appropriate antimicrobial coverage at least one day prior to identification of the organism was associated with improved survival [17]. Furthermore, the pathogen itself carries a prognostic risk factor. Fungal infections carry the lowest survival rates $(17 \%)$, followed by Gram positive (52\%) and Gram-negative $(57 \%)$ bacteria. Survival rate has been reported to be the highest in patients in whom no pathogen was identified $(73 \%)$ [18].

Initially, broad antibiotic coverage is recommended. Neonates are most frequently placed on ampicillin and an aminoglycoside or a $3^{\text {rd }}$ generation cephalosporin as the most common pathogens in this age group include: group B streptococcus, Gram-negative enteric organisms and Listeria "monocytogenes" "meningitidis" and "influenzae". In infants and children over the age of 4-6 weeks, a 3rd generation cephalosporin is the first-line agent as Streptococcus pneumoniae, Neisseria and Heamophilus are the most common pathogens in this age group. In light of the antibiotic resistance of S. pneumoniae and the increasing prevalence of community-acquired, methicillin-resistant Staphylococcus aureus (CA-MRSA), vancomycin should be added as empiric coverage. Finally, in the setting of a high degree of suspicion of Gram negative infection or acquisition of a nosocomial source of sepsis (e.g. Pseudomonas species), additional coverage with an aminoglycoside is recommended.

Because of the broad coverage that includes some anaerobes and low renal toxicity associated with piperacillin/tazobactam, this combination is often chosen for 
empiric administration. The anti-viral agent, acyclovir, should be administered if there is a high clinical suspicion of a herpes viral infection, especially with neonates presenting with fever, seizure, and/or elevated liver enzymes and coagulopathy.

In immunocompetent children the decision to start empiric antifungal therapy remains controversial. In the context in which a child is not improving over the initial days of empiric coverage or there is a higher risk for fungal infection (e.g. presence of indwelling devices, immunosuppression or other significant co-morbidities) then antifungal coverage can be provided. The development of agents equally as effective as amphotericin but with substantially reduced nephrotoxicity such as fluoconazole and capsofungin may ultimately shift the risk/benefit analysis towards more conservative, earlier initiation of empiric anti-fungal coverage in select, high-risk populations. Finally, the ability to limit treatment once the organism has been identified will reduce the number of potential side effects and curtail the development of pathogen resistance related to imprudent use of broad-spectrum antibiotics.

Finally, in the developing world, practitioners must be aware of infectious diseases unique to this setting that can cause severe sepsis and influence management strategies, notably malaria and dengue fever. Recent guidelines from the American College of Critical Care Medicine do not specifically discuss malaria and dengue fever, which have to be included in the differential diagnosis of any child with fever and shock in many regions of Asia and Africa and present unique challenges in the PICU setting [19]. The inclusion of unique infectious diseases in the differential may direct the decision to add metronidazole or clindamycin to empiric antibiotic coverage in high-risk regions [20].

\section{MAINTENANCE OF OXYGEN DELIVERY}

The cornerstone of conventional therapy in sepsis remains the maintenance of adequate oxygen delivery in the face of myocardial depression, capillary leak, acidosis, and massive cytokine release. Adequate oxygen delivery $\left(\mathrm{DO}_{2}\right)$ to maintain tissue oxygenation requires sufficient tissue perfusion and oxygen content as determined by the following equation:

$$
\mathrm{DO}_{2}(\mathrm{~mL} / \mathrm{min})=\mathrm{CO} \times \mathrm{CaO}_{2}=[\mathrm{HR} \times \mathrm{SV}] \times[(1.34 \times \mathrm{Hgb}
$$
$\left.\left.x \mathrm{SaO}_{2}\right)+\left(0.0031 \times \mathrm{PaO}_{2}\right)\right]$

where, $\mathrm{DO}_{2}$ is oxygen delivery, $\mathrm{CaO}_{2}$ is arterial oxygen content, $\mathrm{CO}$ is cardiac output, $\mathrm{SV}$ is stroke volume, $\mathrm{HR}$ is heart rate, and $\mathrm{PaO}_{2}$ is arterial partial oxygen tension. A number of studies of adults in selected patient subpopulations have suggested improved outcomes when achieving supra-normal levels of oxygen delivery [21, 22]. Whether this improves outcomes in children has not been conclusively shown. Ultimately, we may discover this approach to be ineffective as suggested by the observation that septic patients often have a diminished ability to extract oxygen such that increasing oxygen delivery will not improve oxygen extraction at the cellular level. For these reasons the best assessment of adequate oxygen delivery and uptake includes a combination of measures of adequate perfusion (such as lactate level and urine output) in addition to blood pressure and SVC oxygen saturation.
As is evident in calculating the arterial content of oxygen, maintaining an adequate hemoglobin level is an important factor in providing sufficient oxygen delivery. While there is no recommended target hemoglobin level for children, a hemoglobin concentration of $10 \mathrm{~g} / \mathrm{dL}$ has been espoused for adults with cardiopulmonary compromise based on improved outcomes when this goal was achieved as part of EGDT [4]. On the other hand, a study of 637 stable, pediatric intensive care patients who had $\mathrm{Hgb}<9.5 \mathrm{~g} / \mathrm{dL}$ were randomly assigned to a hemoglobin ( $\mathrm{Hgb}$ ) threshold of $7 \mathrm{~g} / \mathrm{dL}$ for red cell transfusion ("restrictive" group) or to a threshold of $9.5 \mathrm{~g} / \mathrm{dL}$ ("liberal" strategy). In the restrictive group, there was $96 \%$ reduction in the number of patients who had any transfusion exposure and a $44 \%$ decrease in the number of red cell transfusions administered [23]. This was accomplished without any increase in the rates of new or progressive multiple organ dysfunction syndrome (MODS) or nosocomial infections and other important clinical parameters [23]. This study only suggests a lower Hgb threshold may be acceptable in a well-defined subset of stable pediatric intensive care patients. Broader extrapolation of these results should be tempered as the study subjects were characterized as less severely ill and did not include unstable patients with septic shock. Thus, it is generally recommended that blood transfusion be considered to aid in achieving a goal of increased $\mathrm{CaO}_{2}$ in the setting of a low $\mathrm{Hgb}$ concentration with targeting of the $\mathrm{SVC} \mathrm{O}_{2}$ saturation to be $>70 \%$.

Ultimately in patients with refractory septic shock, extracorporeal membrane oxygenation (ECMO) may be an important tool to consider for providing adequate oxygen delivery. In a small series, Maclaren et al. reported that the survival rate to hospital discharge for children with septic shock managed with ECMO was $47 \%$ [24]. Because of the retrospective design, it was not possible for these investigators to accurately predict whether the patients might have survived without ECMO. The only way to determine the benefit of ECMO in septic patients is to do a prospective randomized trial such as the CESAR study in adult respiratory failure. Unfortunately, even in the setting of seeming adequate oxygen delivery it is not uncommon to observe altered oxygen utilization by tissues reflected by rising lactates and progressive organ failure despite even full venoarterial ECMO support providing what should be more than sufficient oxygen delivery. Thus, even maximal management of oxygen delivery is insufficient to overcome the pathophysiologic processes mediating altered oxygen uptake. Intensive care medicine awaits the identification of "insulin for oxygen" that can be added to our therapeutic armamentarium that currently remains predominantly directed at oxygen delivery.

\section{MANAGEMENT OF ADDITIONAL END-ORGAN DYSFUNCTION}

As severe sepsis or septic shock proceeds to multiorgan dysfunction, the ability to maintain adequate oxygen delivery is increasingly more difficult.

\section{Respiratory Dysfunction}

Acute lung injury / Acute respiratory distress syndrome $(A L I / A R D S)$ can result from direct toxin action or cytokines released during the host response. Initiation of a lung 
protective ventilation strategy using low lung volumes (less than $12 \mathrm{ml} / \mathrm{kg}$ ) extrapolated from the ARDSnet study is recommended. This multi-institutional trial demonstrated that a targeted tidal volume of $6 \mathrm{ml} / \mathrm{kg}$ of ideal body weight, with limitation of plateau pressure $<30 \mathrm{~cm} \mathrm{H} 2 \mathrm{O}$ decreased the mortality rate to $31 \%$ as compared to the conventional group ventilated with $12 \mathrm{ml} / \mathrm{kg}$ (39.8\% mortality) [25]. Additional adjuvant strategies such as surfactant, inhaled nitric oxide (NO), prone position, steroids and high frequency oscillatory ventilation (HFOV) are other tools available to manage the hypoxia associated with ALI/ARDS. Readers are directed to the following references to review these modalities [26-31].

\section{Endocrine Dysfunction}

A-Glucose metabolism derangements occur frequently in the septic patient characterized by hyperglycemia and insulin resistance. Hyperglycemia is potentially harmful because it impairs neutrophil function, increases risk of infection, impairs wound healing and acts as procoagulant [26]. Insulin can control hyperglycemia and improve lipid levels [27], and may possess anti-inflammatory [28] and anti-coagulant actions. Thus, it was hypothesized that using insulin to more tightly regulate serum glucose levels would clinically benefit critically ill patients. Van den Berghe et al. conducted a study of tight glycemic control in critically ill surgical patients and found that intensive insulin therapy (to maintain blood glucose $80-110 \mathrm{mg} / \mathrm{dL}$ ) as compared to conventional insulin therapy (allowing blood glucose to range from 180$200 \mathrm{mg} / \mathrm{dL}$ ) was associated with decreased ICU mortality (from $8 \%$ to $4.6 \%$ ). It is important to note that their control group had a substantially higher mortality rate $(8 \%)$ than is typically reported for similar ICU patient cohorts. Nevertheless, at their center, tight glycemic control was also associated with decreased in-hospital mortality (by 34\%), blood stream infections (by 46\%) and less prolonged mechanical ventilation and intensive care [29]. A similar study conducted on critically ill medical ICU patients reported that intensive insulin therapy did not decrease overall mortality. Interestingly, with tight glycemic control there was an as yet unexplained increase in mortality in patients who stayed in the ICU less than 3 days while a favorable decrease in mortality was observed in patients who stayed in ICU more than 3 days [30]. Most recently, Brunkhorst et al. conducted a study of tight glycemic control in patients with severe sepsis and reported that intensive insulin therapy was associated with higher rate of hypoglycemia and no significant difference at 28 days mortality or organ dysfunction score as compared to conventional insulin therapy [31].

It is important to contextualize this work historically and appreciate that prior to Van den Berghe's initial report "stress-induced" hyperglycemia was thought of as a normal adaptive response to increased glucose utilization needs of the stressed tissues. In this case, an association between hyperglycemia and mortality would be expected as it would simply be a reflection of severity of illness. In fact, in the only study in pediatrics in which severity of illness was controlled for (using PRISM scoring) no independent association between hyperglycemia and outcomes was observed [32]. Given these data and the heightened risk of hypoglycemia that has been consistently reported in trials of tight glycemic control, no recommendation can be given for this approach in critically ill infants and developing children. Numerous on-going studies should provide more definitive information upon which to base future decisions with regards to the use of intensive insulin therapy in the pediatric patient with sepsis.

$B$-Adrenal insufficiency (AI) is another endocrine dysfunction that has been identified as a complicating factor in sepsis. The role of corticosteroid therapy in the management of septic shock has been extensively debated for many years. Its management is complicated by the myriad of different definitions ("absolute" versus "relative" adrenal insufficiency), using free vs. total cortisol serum levels to define AI and the tissue specific resistance to corticosteroids. Other controversial issues in AI include the debate as to the optimal dose of cosyntropin used in stimulation testing, whether steroids should be replaced at physiologic versus stress versus "shock doses" and what the duration of treatment should be. This lack of clarity is unfortunate, because AI appears to occur frequently in children. Pizarro and co-workers studied 57 children with septic shock and found that the incidence of adrenal insufficiency (AI) overall was 44\% (absolute AI 18\%, relative AI $26 \%$ ) with the highest incidence of mortality among those children whose post-stimulation cortisol level remained $<9 \mu \mathrm{g} / \mathrm{dL}$ [33]. Additional data from patients with meningococcemia reported significantly lower cortisol levels in children who died [34]. However, such observations have not been consistent. For example, in a second study of children with meningococcemia, Hatherill et al. found no relationship between cortisol levels and mortality. Though they did report a need for more vasopressor support in those patients who similarly displayed an inadequate response to cosyntropin stimulation (cut off $<7 \mu \mathrm{g} / \mathrm{dL}$ ) [35]. While the debate continues as to the impact of absolute and/or relative adrenal insufficiency in septic shock, adult colleagues embarked on seminal trials aimed at determining the effect of treating patients with severe sepsis with steroid replacement therapy.

In a now landmark trial, Annane et al. published their results of a randomized controlled study (French Study) in nearly 300 adult patients with septic shock. These investigators found that in catecholamine-dependent septic shock patients with relative adrenal insufficiency, treatment with the combination of hydrocortisone and fludrocortisone was associated with a significant reduction in both 28-day (63\% in placebo vs. $55 \%$ with corticosteroid treatment) and ICU- (70\% vs. 58\%) mortality [36]. Of note, in this study a number of subjects were medicated with etomidate which is now known to influence the adrenocortical axis. In follow up this study, Sprung et al. published the multi-center, randomized, controlled CORTICUS Study which examined $\sim 500$ adult patients with septic shock who received hydrocortisone for 5 days. While there was no significant difference in mortality among patients who received hydrocortisone, regardless of their response to cosyntropin, the duration of time to reversal of shock was significantly shorter among all patients treated with hydrocortisone independent of the results of their cosyntropin test [37]. In addition to there being no mortality benefit, there was an increase prevalence of nosocomial infections, new sepsis and hyperglycemia in the hydrocortisone-treated cohort leading 
the authors of the study to not recommend adjunctive hydrocortisone therapy for adults with septic shock. Though there are a variety of factors that may have contributed to differences among the two large studies (French and CORTICUS), their respective conclusions only add to the complexity of the debate regarding cortisol-replacement therapy in severe sepsis. Complicating this debate is the reality that in pediatrics very few data exist to provide strong evidence for the use of steroids in severe sepsis/septic shock. For additional information on this subject, the reader is directed to a comprehensive and timely summary of these data [38]. In summary, given the dearth of clinical data in pediatrics, it is not possible to recommend routine adjuvant corticosteroid therapy in pediatric sepsis outside of those children at significant risk of absolute adrenal insufficiency (e.g. purpura fulminans, congenital adrenal hyperplasia, active steroid therapy for underlying disease, panhypopituitarism) Outside of these high risk populations, we advise that care providers maintain equipoise in assessing the benefit of adjuvant steroid therapy. At this time, a common approach in managing pediatric sepsis is to consider the use of exogenous steroids in patients with severe sepsis who are "fluid refractory" and on two or more vasoactive agents. This approach generally concurs with the "expert" recommendations from the international task force of the American College of Critical Care Medicine (ACCCM) [37].

\section{Gastrointestinal Dysfunction and Nutritional Support}

Providing adequate nutritional support can be a major challenge, but is of utmost importance in pediatric patients with sepsis. These patients often have poor nutrition prior to admission because of the insidious onset of illness and often may not be fed in the first few days of illness. This state, combined with the increased metabolic rate (due to fever, anxiety, muscle effort, shivering) associated with sepsis, places the septic patient at risk for protein calorie malnutrition. Intestinal hypoperfusion in combination with insufficient local enterocyte nutrition can cause mucosal barrier dysfunction and may contribute to translocation of bacteria and endotoxin from the intestine into the blood stream [39, 40]. While the use of enteral feeding in critical illness has been shown to improve survival and decrease hospital stay [41], it must be balanced with the risk of stressing intestinal function in the face of poor splanchnic perfusion, especially in the presence of vasopressors such as higher-dose (i.e. $\alpha$-adrenergic range) epinephrine and norepinephrine $[42,43]$. A commonly practiced goal is to initiate at least modest volumes of enteral feeds after the first 24 hours of resuscitation and upon restoration of stable hemodynamics provided this is not requiring more than inotropic doses of epinephrine or hormonal replacement dosing levels of vasopressin. Enteral feeds are always preferred over the parenteral route for a number of reasons, including a beneficial trophic effect on intestinal villus (that theoretically reduces bacterial translocation) support of gut associated lymphoid tissue, lower cost, and lower risk of infection associated with delivery as there is no need for central venous access. Regardless of which mode of nutrition is chosen, the goal of achieving nitrogen balance is important for allowing recovery and return to physiologic homeostasis.
Finally, in the absence of enteral feedings, protection from stress-related gastrointestinal ulcer formation is advised.

The issue of initiating enteral feeds in the setting of severe sepsis is more cogent in the developing world where the incidence of severe malnutrition is substantially higher, notably in Africa and Asia. Even in areas of South America clinically notable malnutrition was observed in up to half the PICU patients [44]. As a result, not only are such children more susceptible to sepsis due to associated poor immunologic response, but in those with hypoproteinemia and "edematous" malnutrition, there may be adverse effects related to the type of aggressive fluid resuscitation espoused in the protocols directing care in the developed world. As a result, the World Health Organization recommends the use of small fluid boluses and thereafter use of blood transfusions for fluid resuscitation in severely malnourished children with severe sepsis [45]. Such factors related to geographical areas with limited health care resources led to the creation of the World Federation of Pediatric Intensive and Critical Care Societies (WFPICCS; www.wfpiccs.org) which is a global educational, interactive and participative program to assist in treating sepsis and septic shock with such unique challenges posed by the health care environment.

\section{Renal Dysfunction}

Acute renal failure (ARF) is a complication that occurs in one-quarter of patients with severe sepsis and up to onehalf of patients with septic shock [46]. Development of ARF poses two primary challenges: clearance dysfunction and fluid overload; and frequently necessitates the institution of renal replacement therapy. Recently, a number of pediatric trials have examined the effect of ARF and the consequent need for Continuous Renal Replacement Therapy (CCRT) on clinical outcomes. For example, Goldstein and coworkers performed a single center study which suggested that increased fluid overload (FO) status at CRRT initiation was associated with an increased mortality; irrespective of severity of illness as measured by PRISM II score [47]. In a subsequent prospective, multicenter study of 116 pediatric patients with MODS, the percent FO (calculated as: $[\% \mathrm{FO}$ (Fluid overload $)=($ Fluid In-Fluid Out $) /($ PICU admission weight) $\times 100 \%]$ affected survival which was significantly better for patients with $<20 \%$ FO $(58 \%)$ versus $>20 \%$ FO $(40 \%)$ at CRRT initiation $(\mathrm{p}<0.002)$ [48].

Foland and coworkers similarly reported that patients with multiple organ failure who received CRRT when they were $<10 \%$ FO had better outcomes than those who were $>10 \%$ fluid overloaded [49]. We caution judicious interpretation of these data recognizing the possibility that FO may simply be a marker of illness severity and endothelial dysfunction rather than a causative factor of organ failure/death. Nevertheless, the results suggest that earlier initiation of CRRT in centers that possess this capability should be considered. Advantages to CRRT are that it affords for provision of adequate caloric intake without exacerbating fluid overload and that it may facilitate cytokine or other harmful mediator removal during the acute phase of sepsis which may aid in stabilizing the respiratory and cardiovascular physiology, though this latter hypothesis remains to be fully tested. Thus, we advocate the use of 
CRRT in adequately resuscitated, hemodynamically stable patients who have renal insufficiency or failure such that adequate support (nutrition, drips, etc) cannot be provided without exacerbating fluid overload. The application of adjuvant extracorporeal therapies such as endotoxin removal and/or plasmapharesis/plasma exchange is also easy to convert to in the setting of established CRRT, but whether these therapies afford any benefit remains anecdotal and speculative.

\section{CNS/PNS Dysfunction}

Patients may present with alterations in mental status upon presentation with sepsis, which may be explained by hypoxia, hypoperfusion, direct bacterial infection or indirect toxin effects on neurons. This pathophysiology is further exacerbated by therapeutic interventions including sedative use (for intubation, to decrease work of breathing and to decrease oxygen consumption) and neuromuscular blocking agents. Making a conservative assumption that the septic patient may have a component of cerebral edema, it is recommended that isotonic fluid be used as maintenance fluid and that the head of bed be raised to $30-45^{\circ}$ to enhance cerebral venous return, and parenthetically reduce aspiration/ventilator associated pneumonia (VAP) risk.

In addition to CNS dysfunction, there is a spectrum of conditions that affect critically ill patients called Critical Illness Polyneuropathy and Myopathy (CIPNM). Hyperosmolality, parenteral nutrition, use of neuromuscular blocking agents (NMBAs), and Glasgow Coma Scale score $<10$ may be independent risk factors for the development of CIPNM in septic patients [50]. Thus, one should aim to minimize the use of NMBAs, and if used clinicians should consider instituting a daily drug holiday protocol during which the patient's neuromuscular status is assessed and NMBAs weaned to the minimal effective dose. It is also recommended to minimize the use of corticosteroids, aminoglycosides and furosemide simultaneously as these medications may similarly affect in the neuromuscular axis.

\section{Hematological Dysfunction}

Numerous hematologic abnormalities can develop in the setting of sepsis, but a common pathophysiologic alteration is the change from an anti-coagulant to a pro-coagulant intravascular phenotype. As this was in part associated to decreased circulating levels of activated Protein C (APC), treatment with a recombinant form of this protein in adults with severe sepsis was initiated and shown to decrease mortality in those patients with the highest quartile risk of mortality (as defined by APACHE scoring). Unfortunately, a related study of APC administration in children with severe sepsis showed no benefit and a potential increased risk of cerebral bleeding as reported in the RESOLVE trial [51].

\section{SUMMARY}

Sepsis remains one of the most pressing clinical challenges for the pediatric intensivists in both the developed and developing countries. It is apparent that while a great deal is now understood about the biological and molecular mechanisms involved in sepsis, this knowledge has yet to be translated into newer innovative strategies. At present, therapeutic modalities for sepsis remain largely supportive and founded on the fundamental physiologic principles of early and aggressive resuscitation, provision of adequate oxygen delivery, pathogen eradication and supportive care of multi-organ failure. Certainly, with this approach, mortality in pediatric sepsis has improved over the past decades. However, the fact that over 4,000 children die in the U.S. each year with severe sepsis and the additional incompletely defined, but exceptional burden of pediatric sepsis worldwide demand that further advances be made. Realization of this goal requires investigators committed to achieving further mechanistic insights into the physiologic, molecular, and genetic biology of sepsis, complimented by large scale, pediatric-specific interventional trials and establishment of novel, non-conventional strategies.

\section{ABBREVIATIONS}

$($ EGDT) = Early goal-directed therapy

$\left(\mathrm{SVC} \mathrm{O}_{2}\right)=$ Superior vena caval oxygen saturation

$(\mathrm{SVR}) \quad=$ Systemic vascular resistance

$(\mathrm{CVP})=$ Central venous pressure

(MODS)

$\left(\mathrm{CaO}_{2}\right)=$ arterial oxygen content

$(\mathrm{Hgb}) \quad=$ Hemoglobin

$(\mathrm{ECMO})=$ Extracorporeal membrane oxygenation

$(\mathrm{ALI} / \mathrm{ARDS})=$ Acute lung injury $/$ acute respiratory distress syndrome

$(\mathrm{ARF}) \quad=$ Acute renal failure

$(\mathrm{CCRT})=$ Continuous renal replacement therapy

(FO) $\quad=$ Fluid overload

\section{REFERENCES}

[1] Watson RS, Linde-Zwirble WT , Carcillo JA, Angus DC. Severe sepsis in children: A U.S. epidemiologic survey. Crit Care Med 2001; 28: A46.

[2] Watson RS, Linde-Zwirble WT, Lidicker J, Carcillo JA, Angus DC. The increasing burden of severe sepsis in U.S. children. Crit Care Med 2002; 29: A8.

[3] Odetola FO, Gebremariam A, Freed GL. Patient and hospital correlates of clinical outcomes and resource utilization in severe pediatric sepsis. Pediatrics 2007; 119(3): 487-94.

[4] Rivers E, Nguyen B, Havstad S, et al. Early goal-directed therapy in the treatment of severe sepsis and septic shock. N Engl J Med 2001; 345(19): 1368-77

[5] Kissoon N. Sepsis and septic shock. A global perspective and initiative. Saudi Med J. 2008; 29(10): 1383-7.

[6] Booy R, Habibi P, Nadel S, et al. Reduction in case fatality rate from meningococcal disease associated with improved healthcare delivery. Arch Dis Child 2001; 85(5): 386-90.

[7] Brierley J, Carcillo JA, Choong K, et al. Clinical practice parameters for hemodynamic suppor of pediatric and neonatal septic shock: 2007 update from the American College of Critical Care Medicine. Crit Care Med 2009; 37(2): 666-88.

[8] de Oliveira CF, de Oliveira DS, Gottschald AF, et al. ACCM/PALS haemodynamic support guidelines for paediatric septic shock: an outcomes comparison with and without monitoring central venous oxygen saturation. Intensive Care Med 2008; 34(6): 1065-75.

[9] Finfer S, Bellomo R, Boyce N, French J, Myburgh J, Norton R. A comparison of albumin and saline for fluid resuscitation in the intensive care unit. N Engl J Med 2004 27; 350(22): 2247-56. 
[10] Maitland K, Pamba A, English M, et al. Randomized trial of volume expansion with albumin or saline in children with severe malaria: preliminary evidence of albumin benefit. Clin Infect Dis 2005; 40(4): 538-45.

[11] Sookhai S, Wang JH, Winter D, Power C, Kirwan W, Redmond HP. Dopamine attenuates the chemoattractant effect of interleukin8: a novel role in the systemic inflammatory response syndrome. Shock 2000; 14(3): 295-9.

[12] Schilling T, Grundling M, Strang CM, Moritz KU, Siegmund W, Hachenberg T. Effects of dopexamine, dobutamine or dopamine on prolactin and thyreotropin serum concentrations in high-risk surgical patients. Intensive Care Med 2004; 30(6): 1127-33.

[13] Ceneviva G, Paschall JA, Maffei F, Carcillo JA. Hemodynamic support in fluid-refractory pediatric septic shock. Pediatrics 1998; 102(2): e19.

[14] Mizock BA, Falk JL. Lactic acidosis in critical illness. Crit Care Med $1992 ; 20(1)$ : 80-93.

[15] Bassi G, Radermacher P, Calzia E. Catecholamines and vasopressin during critical illness. Endocrinol Metab Clin North Am 2006; 35(4): 839-57.

[16] Schrier RW, Wang W. Acute renal failure and sepsis. N Engl J Med $2004 ; 351(2): 159-69$.

[17] Bryan CS, Reynolds KL, Brenner ER. Analysis of 1,186 episodes of gram-negative bacteremia in non- university hospitals: the effects of antimicrobial therapy. Rev Infect Dis 1983; 5(4): 629-38.

[18] Glauser MP, Zanetti G, Baumgartner JD, Cohen J. Septic shock: pathogenesis. Lancet 1991; 338(8769): 732-6.

[19] Singhi S, Kissoon N, Bansal A. Dengue and dengue hemorrhagic fever: management issues in an intensive care unit. J Pediatr (Rio J) 2007; 83(2 Suppl): S22-35.

[20] Singhi S, Kissoon NN. Pediatric intensive care in India: why, how and role of global collaboration! Indian J Pediatr 2008; 75(6): 58990 .

[21] Gattinoni L, Brazzi L, Pelosi P, et al. A trial of goal-oriented hemodynamic therapy in critically ill patients. SvO2 Collaborative Group. N Engl J Med 1995; 333(16): 1025-32.

[22] Kern JW, Shoemaker WC. Meta-analysis of hemodynamic optimization in high-risk patients. Crit Care Med 2002; 30(8): 1686-92.

[23] Lacroix J, Hebert PC, Hutchison JS, et al. Transfusion strategies for patients in pediatric intensive care units. N Engl J Med 2007; 356(16): 1609-19.

[24] Maclaren G, Butt W, Best D, Donath S, Taylor A. Extracorporeal membrane oxygenation for refractory septic shock in children: one institution's experience. Pediatr Crit Care Med 2007; 8(5): 447-51.

[25] Ventilation with lower tidal volumes as compared with traditional tidal volumes for acute lung injury and the acute respiratory distress syndrome. The Acute Respiratory Distress Syndrome Network. N Engl J Med 2000; 342(18): 1301-8.

[26] Carr ME. Diabetes mellitus: a hypercoagulable state. J Diabetes Complications 2001; 15(1): 44-54.

[27] Mesotten D, Swinnen JV, Vanderhoydonc F, Wouters PJ, Van den Berghe G. Contribution of circulating lipids to the improved outcome of critical illness by glycemic control with intensive insulin therapy. J Clin Endocrinol Metab 2004; 89(1): 219-26.

[28] Dandona P, Aljada A, Mohanty $\mathrm{P}$, et al. Insulin inhibits intranuclear nuclear factor kappaB and stimulates IkappaB in mononuclear cells in obese subjects: evidence for an antiinflammatory effect? J Clin Endocrinol Metab 2001; 86(7): 325765

[29] van den Berghe G, Wouters P, Weekers F, et al. Intensive insulin therapy in the critically ill patients. N Engl J Med 2001; 345(19): 1359-67.

[30] Van den Berghe G, Wilmer A, Hermans G, et al. Intensive insulin therapy in the medical ICU. N Engl J Med 2006; 354(5): 449-61.

[31] Brunkhorst FM, Engel C, Bloos F, et al. Intensive insulin therapy and pentastarch resuscitation in severe sepsis. N Engl J Med 2008; 358(2): 125-39.
[32] Klein GW, Hojsak JM, Schmeidler J, Rapaport R. Hyperglycemia and outcome in the pediatric intensive care unit. J Pediatr 2008; 153(3): 379-84.

[33] Pizarro CF, Troster EJ, Damiani D, Carcillo JA. Absolute and relative adrenal insufficiency in children with septic shock. Crit Care Med 2005; 33(4): 855-9.

[34] Riordan FA, Thomson AP, Ratcliffe JM, Sills JA, Diver MJ, Hart CA. Admission cortisol and adrenocorticotrophic hormone levels in children with meningococcal disease: evidence of adrenal insufficiency? Crit Care Med 1999; 27(10): 2257-61.

[35] Hatherill M, Tibby SM, Hilliard T, Turner C, Murdoch IA. Adrenal insufficiency in septic shock. Arch Dis Child 1999; 80(1): 51-5.

[36] Annane D, Sebille V, Charpentier C, et al. Effect of treatment with low doses of hydrocortisone and fludrocortisone on mortality in patients with septic shock. JAMA 2002; 288(7): 862-71

[37] Marik PE, Pastores SM, Annane D, et al. Recommendations for the diagnosis and management of corticosteroid insufficiency in critically ill adult patients: consensus statements from an international task force by the American College of Critical Care Medicine. Crit Care Med 2008; 36(6): 1937-49.

[38] Zimmerman JJ. A history of adjunctive glucocorticoid treatment for pediatric sepsis: moving beyond steroid pulp fiction toward evidence-based medicine. Pediatr Crit Care Med. 2007; 8(6): 5309.

[39] Crouser ED, Dorinsky PM. Gastrointestinal tract dysfunction in critical illness: pathophysiology and interaction with acute lung injury in adult respiratory distress syndrome/multiple organ dysfunction syndrome. New Horiz 1994; 2(4): 476-87.

[40] Swank GM, Deitch EA. Role of the gut in multiple organ failure: bacterial translocation and permeability changes. World J Surg 1996; 20(4): 411-7.

[41] Marik PE, Zaloga GP. Early enteral nutrition in acutely ill patients: a systematic review. Crit Care Med 2001; 29(12): 2264-70.

[42] Marik PE, Mohedin M. The contrasting effects of dopamine and norepinephrine on systemic and splanchnic oxygen utilization in hyperdynamic sepsis. JAMA 1994; 272(17): 1354-7.

[43] Meier-Hellmann A, Reinhart K, Bredle DL, Specht M, Spies CD, Hannemann L. Epinephrine impairs splanchnic perfusion in septic shock. Crit Care Med 1997; 25(3): 399-404.

[44] Delgado AF, Okay TS, Leone C, Nichols B, Del Negro GM, Vaz FA. Hospital malnutrition and inflammatory response in critically ill children and adolescents admitted to a tertiary intensive care unit. Clinics 2008; 63(3): 357-62.

[45] Organization WH. Management of Severe Malnutrition: A Manual for Physicians and Other Senior Health Workers. Geneva: WHO 1999.

[46] Rangel-Frausto MS, Pittet D, Costigan M, Hwang T, Davis CS, Wenzel RP. The natural history of the systemic inflammatory response syndrome (SIRS). A prospective study. JAMA 1995; 273(2): 117-23.

[47] Goldstein SL, Currier H, Graf C, Cosio CC, Brewer ED, Sachdeva R. Outcome in children receiving continuous venovenous hemofiltration. Pediatrics 2001; 107(6): 1309-12.

[48] Goldstein SL, Somers MJ, Baum MA, et al. Pediatric patients with multi-organ dysfunction syndrome receiving continuous renal replacement therapy. Kidney Int 2005; 67(2): 653-8.

[49] Foland JA, Fortenberry JD, Warshaw BL, et al. Fluid overload before continuous hemofiltration and survival in critically ill children: a retrospective analysis. Crit Care Med 2004; 32(8): 1771-6.

[50] Garnacho-Montero J, Madrazo-Osuna J, Garcia-Garmendia JL, et al. Critical illness polyneuropathy: risk factors and clinical consequences. A cohort study in septic patients. Intensive Care Med 2001; 27(8): 1288-96.

[51] Nadel S, Goldstein B, Williams MD, et al. Drotrecogin alfa (activated) in children with severe sepsis: a multicentre phase III randomised controlled trial. Lancet 2007; 369(9564): 836-43.

This is an open access article licensed under the terms of the Creative Commons Attribution Non-Commercial License (http://creativecommons.org/licenses/by-nc/3.0/) which permits unrestricted, non-commercial use, distribution and reproduction in any medium, provided the work is properly cited. 